EPJ Web of Conferences 71, 00129 (2014)

DOI: 10.1051/epjconf/20147100129

(C) Owned by the authors, published by EDP Sciences, 2014

\title{
Analytical relation between quark confinement and chiral sym- metry breaking in odd-number lattice QCD
}

\author{
Hideo Suganuma ${ }^{1}$, a , Takahiro M. Doi ${ }^{1}$, and Takumi Iritani ${ }^{2}$, \\ ${ }^{1}$ Department of Physics \& Division of Physics and Astronomy, Graduate School of Science, \\ Kyoto University, Kitashirakawaoiwake, Sakyo, Kyoto 606-8502, Japan \\ ${ }^{2}$ High Energy Accelerator Research Organization (KEK), Tsukuba, Ibaraki 305-0801, Japan
}

\begin{abstract}
To clarify the relation between confinement and chiral symmetry breaking in QCD, we consider a temporally odd-number lattice, with the temporal lattice size $N_{t}$ being odd. We here use an ordinary square lattice with the normal (nontwisted) periodic boundary condition for link-variables in the temporal direction. By considering $\operatorname{Tr}\left(\hat{U}_{4} \hat{D}^{N_{t}-1}\right)$, we analytically derive a gauge-invariant relation between the Polyakov loop $\left\langle L_{P}\right\rangle$ and the Dirac eigenvalues $\lambda_{n}$ in QCD, i.e., $\left\langle L_{P}\right\rangle \propto \sum_{n} \lambda_{n}^{N_{t}-1}\left\langle n\left|\hat{U}_{4}\right| n\right\rangle$, which is a Dirac spectral representation of the Polyakov loop in terms of Dirac eigenmodes $|n\rangle$. Owing to the factor $\lambda_{n}^{N_{t}-1}$ in the Dirac spectral sum, this relation generally indicates fairly small contribution of low-lying Dirac modes to the Polyakov loop, while the low-lying Dirac modes are essential for chiral symmetry breaking. Also in lattice QCD calculations in both confined and deconfined phases, we numerically confirm the analytical relation, non-zero finiteness of $\left\langle n\left|\hat{U}_{4}\right| n\right\rangle$ for each Dirac mode, and negligibly small contribution from low-lying Dirac modes to the Polyakov loop, i.e., the Polyakov loop is almost unchanged even by removing low-lying Dirac-mode contribution from the QCD vacuum generated by lattice QCD simulations. We thus conclude that low-lying Dirac modes are not essential modes for confinement, which indicates no direct one-to-one correspondence between confinement and chiral symmetry breaking in QCD.
\end{abstract}

\section{Introduction}

Color confinement and spontaneous chiral-symmetry breaking [1] are the two outstanding nonperturbative phenomena in quantum chromodynamics (QCD), and they have been studied as important unsolved subjects in theoretical physics. In particular, to clarify their precise relation is one of the challenging important issues [2-10], and their relation is not yet clarified directly from QCD.

For quark confinement, the Polyakov loop $\left\langle L_{P}\right\rangle$ is one of the typical order parameters, and relates to the single-quark free energy $E_{q}$ as $\left\langle L_{P}\right\rangle \propto e^{-E_{q} / T}$ at temperature $T$. The Polyakov loop is the order parameter of spontaneous breaking of the $Z_{N_{c}}$ center symmetry in QCD [11]. Also, its fluctuation is recently found to be important in the QCD phase transition [12].

For spontaneous chiral-symmetry breaking, the standard order parameter is the quark condensate $\langle\bar{q} q\rangle$, and low-lying Dirac modes are known to be essential, as the Banks-Casher relation shows [13].

ae-mail: suganuma@scphys.kyoto-u.ac.jp

This is an Open Access article distributed under the terms of the Creative Commons Attribution License 2.0, which permits unrestricted use, distribution, and reproduction in any medium, provided the original work is properly cited. 
There are several circumstantial evidence of correlation between confinement and chiral symmetry breaking. For example, lattice QCD simulations have suggested almost coincidence between deconfinement and chiral-restoration temperatures $[11,14]$, although slight difference of about $25 \mathrm{MeV}$ between them is pointed out in some recent lattice QCD studies [15]. Their correlation is also suggested in terms of QCD-monopoles [2, 3], which topologically appear in QCD in the maximally Abelian gauge [16-19], leading to the dual-superconductor picture [20]. As schematically shown in Fig.1, confinement and chiral symmetry breaking are simultaneously lost in lattice QCD, by removing the monopoles from the QCD vacuum [3]. This means a crucial role of QCD-monopoles to both confinement and chiral symmetry breaking, so that these two phenomena seem to be related through the monopole. As a possibility, however, to remove the monopoles may be "too fatal" for nonperturbative properties. If this is the case, nonperturbative phenomena are simultaneously lost by their removal.

In fact, if only the relevant ingredient of chiral symmetry breaking is carefully removed from the QCD vacuum, how will be quark confinement?

To obtain the answer, we perform a direct investigation between confinement and chiral symmetry breaking, using the Dirac-mode expansion and projection [6-8].
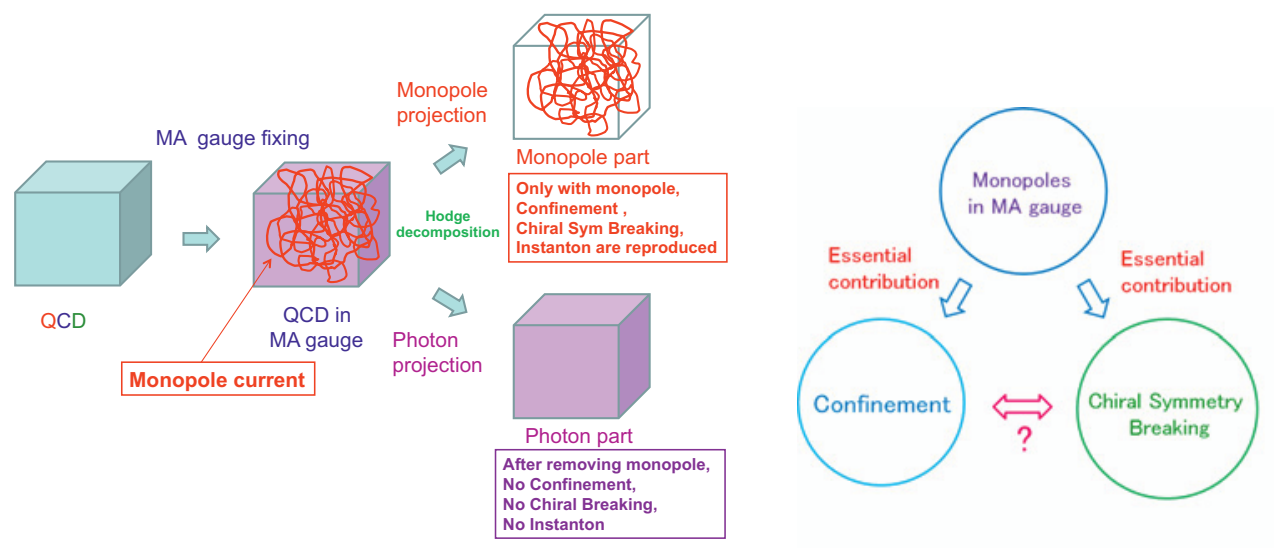

Figure 1. The role of QCD-monopoles to nonperturbative QCD. In the MA gauge, QCD becomes Abelian-like owing to a large effective mass $(\simeq 1 \mathrm{GeV})$ of off-diagonal gluons [18], and QCD-monopoles topologically appear as $\Pi_{2}\left(\mathrm{SU}\left(N_{c}\right) / \mathrm{U}(1)^{N_{c}-1}\right)=\mathbf{Z}^{N_{c}-1}[16,19]$. By the Hodge decomposition, the QCD vacuum can be divided into the monopole part and the photon part. The monopole part has confinement [17], chiral symmetry breaking [3] and instantons [21], while the photon part does not have all of them, as lattice QCD studies show. In spite of the essential role of monopoles, the direct relation of confinement and chiral symmetry breaking is still unclear.

In our previous works, we investigated the relation between confinement and chiral symmetry breaking in more direct manner [6-8] by analyzing confinement in terms of Dirac eigenmodes in QCD, because of the essential role of low-lying Dirac modes for chiral symmetry breaking [13]. Using completeness of the Dirac-mode basis, we proposed "Dirac-mode expansion" and "Dirac-mode projection" to a restricted Dirac-mode space, and investigated the role of low-lying Dirac modes to confinement in SU(3) lattice QCD [6-8]. As the remarkable facts, even by the removal of the coupling to low-lying Dirac modes, we numerically obtained the following lattice-QCD results:

- The Wilson loop obeys the area law, which means a linear quark confinement potential $[6,7]$.

- The slope parameter, i.e., the string tension or the confining force, is almost unchanged [6,7].

- The Polyakov loop remains to be almost zero, which means $Z_{3}$-unbroken confinement phase [8]. 
Thus, quark confinement properties are almost kept even in the absence of low-lying Dirac modes. (Also, "hadrons" appear without low-lying Dirac modes [22], suggesting survival of confinement.) In our studies, we just consider the mathematical expansion by eigenmodes of the Dirac operator $\not D=\gamma_{\mu} D_{\mu}$. For eigenmode expansions, one can deal with any (anti)hermite operator, e.g., $D^{2}=D_{\mu} D_{\mu}$. However, to link with chiral symmetry breaking, we adopt $\not D$ and the expansion by its eigenmodes.

In this study, we consider temporally odd-number lattice QCD, where the temporal lattice size is odd-number, and derive an analytical relation between the Polyakov loop and the Dirac modes. Based on the analytical formula, we discuss the relation between confinement and chiral symmetry breaking.

\section{Lattice QCD formalism}

In this section, we exhibit the mathematical conditions of lattice QCD formalism adopted in this study. We use an ordinary square lattice with spacing $a$ and size $N_{s}^{3} \times N_{t}$, and impose the normal (nontwisted) periodic boundary condition for the link-variable $U_{\mu}(s)=\mathrm{e}^{i a g A_{\mu}(s)}$ in the temporal direction. $\left(A_{\mu}(s)\right.$ is the gluon field, $g$ the gauge coupling, and $s$ the site.) This temporal periodicity is physically required at finite temperature. As the gauge group, we here take $\mathrm{SU}\left(N_{c}\right)$ with $N_{c}$ being the color number. However, arbitrary gauge group $G$ can be taken for most arguments in this paper.

\subsection{Dirac operator, Dirac eigenvalues and Dirac modes in lattice QCD}

On lattices, the Dirac operator $\not D=\gamma_{\mu} D_{\mu}$ is written with $U_{\mu}(s)=\mathrm{e}^{i a g A_{\mu}(s)}$ and $U_{-\mu}(s) \equiv U_{\mu}^{\dagger}(s-\hat{\mu})$ as

$$
\not D_{s, s^{\prime}} \equiv \frac{1}{2 a} \sum_{\mu=1}^{4} \gamma_{\mu}\left[U_{\mu}(s) \delta_{s+\hat{\mu}, s^{\prime}}-U_{-\mu}(s) \delta_{s-\hat{\mu}, s^{\prime}}\right] .
$$

Here, $\hat{\mu}$ is $\mu$-directed vector with $|\hat{\mu}|=a$. Adopting hermite $\gamma$-matrices as $\gamma_{\mu}^{\dagger}=\gamma_{\mu}$, the Dirac operator $\not D$ is anti-hermite and satisfies $\not_{s^{\prime}, s}^{\dagger}=-\not D_{s, s^{\prime}}$. We introduce the normalized Dirac eigen-state $|n\rangle$ as

$$
\not D|n\rangle=i \lambda_{n}|n\rangle, \quad\langle m \mid n\rangle=\delta_{m n}
$$

with the Dirac eigenvalue $i \lambda_{n}\left(\lambda_{n} \in \mathbf{R}\right)$. Due to $\left\{\gamma_{5}, \not D\right\}=0$, the state $\gamma_{5}|n\rangle$ is also an eigen-state of $\not D$ with the eigenvalue $-i \lambda_{n}$. Here, the Dirac eigen-state $|n\rangle$ satisfies the completeness of

$$
\sum_{n}|n\rangle\langle n|=1
$$

For the Dirac eigenfunction $\psi_{n}(s) \equiv\langle s \mid n\rangle$, the explicit form of the Dirac eigenvalue equation $\not D \psi_{n}(s)=i \lambda_{n} \psi_{n}(s)$ is expressed in lattice QCD as

$$
\frac{1}{2 a} \sum_{\mu=1}^{4} \gamma_{\mu}\left[U_{\mu}(s) \psi_{n}(s+\hat{\mu})-U_{-\mu}(s) \psi_{n}(s-\hat{\mu})\right]=i \lambda_{n} \psi_{n}(s) .
$$

The Dirac eigenfunction $\psi_{n}(s)$ can be numerically obtained in lattice QCD, besides a phase factor. By the gauge transformation of $U_{\mu}(s) \rightarrow V(s) U_{\mu}(s) V^{\dagger}(s+\hat{\mu}), \psi_{n}(s)$ is gauge-transformed as

$$
\psi_{n}(s) \rightarrow V(s) \psi_{n}(s)
$$

which is the same as that of the quark field. (To be strict, there can appear an irrelevant $n$-dependent global phase factor $e^{i \varphi_{n}[V]}$, according to arbitrariness of the phase in the basis $|n\rangle$ [7].)

Note here that the spectral density $\rho(\lambda)$ of the Dirac operator $\not D$ relates to chiral symmetry breaking. For example, the Banks-Casher relation [13] shows that the quark condensate $\langle\bar{q} q\rangle$ originates from the zero-eigenvalue density $\rho(0)$ in the limit of large space-time volume $V_{\text {phys }}$ and in the chiral limit, i.e., $\langle\bar{q} q\rangle=-\lim _{m \rightarrow 0} \lim _{V_{p h y s} \rightarrow \infty} \pi \rho(0)$. In fact, the low-lying Dirac modes can be regarded as the essential modes for spontaneous chiral-symmetry breaking in QCD. 


\subsection{Operator formalism in lattice QCD}

In this subsection, we present the operator formalism in lattice QCD [6-8]. We first introduce the link-variable operator $\hat{U}_{ \pm \mu}$ defined by the matrix element of

$$
\left\langle s\left|\hat{U}_{ \pm \mu}\right| s^{\prime}\right\rangle=U_{ \pm \mu}(s) \delta_{s \pm \hat{\mu}, s^{\prime}}
$$

The Dirac operator and the covariant derivative are simply written with the link-variable operator as

$$
\hat{\not D}=\frac{1}{2 a} \sum_{\mu=1}^{4} \gamma_{\mu}\left(\hat{U}_{\mu}-\hat{U}_{-\mu}\right), \quad \hat{D}_{\mu}=\frac{1}{2 a}\left(\hat{U}_{\mu}-\hat{U}_{-\mu}\right)
$$

Also, the Polyakov loop $\left\langle L_{P}\right\rangle$ is simply expressed as the functional trace of $\hat{U}_{4}^{N_{t}}$,

$$
\left\langle L_{P}\right\rangle=\frac{1}{N_{c} V}\left\langle\operatorname{Tr}_{c}\left\{\hat{U}_{4}^{N_{t}}\right\}\right\rangle=\frac{1}{N_{c} V}\left\langle\sum_{s} \operatorname{tr}_{c}\left(\prod_{n=0}^{N_{t}-1} U_{4}(s+n \hat{t})\right)\right\rangle,
$$

with the four-dimensional lattice volume $V \equiv N_{s}^{3} \times N_{t}$ and $\hat{t}=\hat{4}$. Here, " $\operatorname{Tr}_{c}$ " denotes the functional trace of $\operatorname{Tr}_{c} \equiv \sum_{s} \operatorname{tr}_{c}$ including the trace $\operatorname{tr}_{c}$ over color index.

The Dirac-mode matrix element of the link-variable operator $\hat{U}_{\mu}$ can be expressed with $\psi_{n}(s)$ as

$$
\left\langle m\left|\hat{U}_{\mu}\right| n\right\rangle=\sum_{s}\langle m \mid s\rangle\left\langle s\left|\hat{U}_{\mu}\right| s+\hat{\mu}\right\rangle\langle s+\hat{\mu} \mid n\rangle=\sum_{s} \psi_{m}^{\dagger}(s) U_{\mu}(s) \psi_{n}(s+\hat{\mu}) .
$$

Note that the matrix element is gauge invariant [7] due to the gauge transformation property (5),

$$
\begin{aligned}
\left\langle m\left|\hat{U}_{\mu}\right| n\right\rangle & \rightarrow \sum_{s} \psi_{m}^{\dagger}(s) V^{\dagger}(s) \cdot V(s) U_{\mu}(s) V^{\dagger}(s+\hat{\mu}) \cdot V(s+\hat{\mu}) \psi_{n}(s+\hat{\mu}) \\
& =\sum_{s} \psi_{m}^{\dagger}(s) U_{\mu}(s) \psi_{n}(s+\hat{\mu})=\left\langle m\left|\hat{U}_{\mu}\right| n\right\rangle .
\end{aligned}
$$

To be strict, an irrelevant $n$-dependent global phase factor can appear according to the arbitrariness of the phase in the basis $|n\rangle$. However, this phase factor exactly cancels as $e^{i \varphi_{n}} e^{-i \varphi_{n}}=1$ between $|n\rangle$ and $\langle n|$, and does not appear for physical quantities such as the Wilson loop and the Polyakov loop [7].

\section{Derivation of an analytical relation between the Polyakov loop and Dirac modes in temporally odd-number lattice QCD}

In this section, we consider temporally odd-number lattice QCD [9, 10], with the temporal lattice size $N_{t}$ being odd, as shown in Fig.2. Apart from the odd-number $N_{t}$, all the lattice conditions are ordinary. In fact, we use an ordinary square lattice and the normal (nontwisted) periodic boundary condition for the link-variable $U_{\mu}(s)$ in the temporal direction. The spatial lattice size $N_{s}$ is taken to be larger than $N_{t}$, i.e., $N_{s}>N_{t}$. Note that, in the continuum limit of $a \rightarrow 0$ and $N_{t} \rightarrow \infty$, any number of large $N_{t}$ gives the same physical result. Hence, it is no problem to use the odd-number lattice.

As a general mathematical argument of the Elitzur theorem [11], only gauge-invariant quantities such as closed loops and the Polyakov loop survive in QCD. In fact, all the non-closed lines are gaugevariant and their expectation values are zero. Note here that any closed loop needs even-number link-variables on the square lattice, except for the Polyakov loop. (See Fig.2.)

In this temporally odd-number lattice QCD, we consider the following functional trace $[9,10]$ :

$$
I \equiv \operatorname{Tr}_{c, \gamma}\left(\hat{U}_{4} \hat{D}^{N_{t}-1}\right)
$$



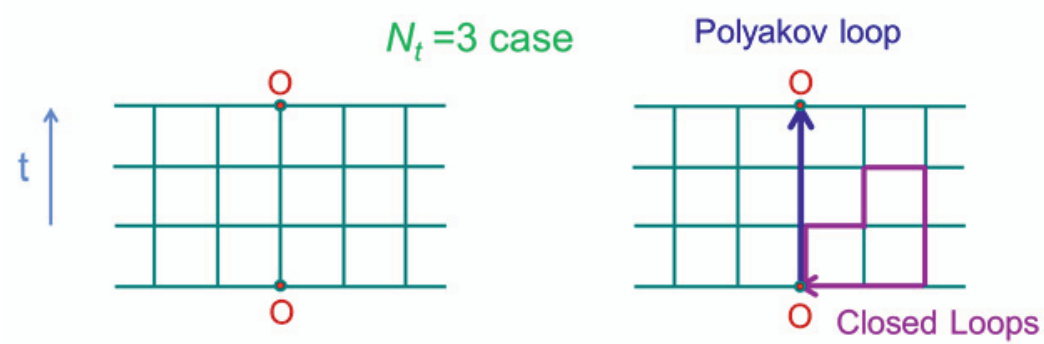

Figure 2. An example of the temporally odd-number lattice $\left(N_{t}=3\right.$ case). Only gauge-invariant quantities such as closed loops and the Polyakov loop survive or do not vanish in QCD, after taking the expectation value, i.e., the gauge-configuration average. Geometrically, closed loops have even-number links on the square lattice.

Here, $\operatorname{Tr}_{c, \gamma} \equiv \sum_{s} \operatorname{tr}_{c} \operatorname{tr}_{\gamma}$ includes $\operatorname{tr}_{c}$ and the trace $\operatorname{tr}_{\gamma}$ over spinor index. Its expectation value

$$
\langle I\rangle=\left\langle\operatorname{Tr}_{c, \gamma}\left(\hat{U}_{4} \hat{D}^{N_{t}-1}\right)\right\rangle
$$

is obtained as the gauge-configuration average in lattice QCD. When the volume $V$ is enough large, one can expect $\langle\hat{O}\rangle \simeq \operatorname{Tr} \hat{O} / \operatorname{Tr} 1$ for any operator $\hat{O}$ even in each gauge configuration.

From Eq.(7), $\hat{U}_{4} \hat{D}^{N_{t}-1}$ can be expressed as a sum of products of $N_{t}$ link-variable operators, because the Dirac operator $\hat{D}$ includes one link-variable operator in each direction of $\pm \mu$. In fact, $\hat{U}_{4} \hat{D}^{N_{t}-1}$ includes "many trajectories" with the total length $N_{t}$ (in the lattice unit) on the square lattice, as shown in Fig.3. Note that all the trajectories with the odd-number length $N_{t}$ cannot form a closed loop on the square lattice, and thus give gauge-variant contribution, except for the Polyakov loop.

$$
N_{t}=3 \text { case }
$$
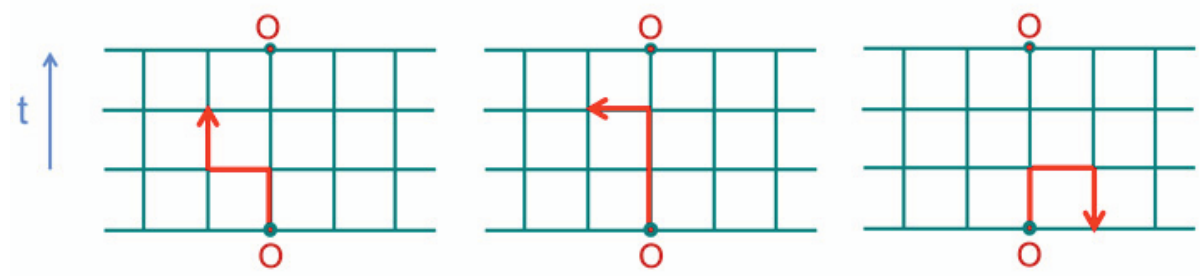

Gauge-variant

Figure 3. Partial examples of the trajectories stemming from $\left\langle\operatorname{Tr}_{c, \gamma}\left(\hat{U}_{4} \hat{D}^{N_{t}-1}\right)\right\rangle$. For each trajectory, the total length is $N_{t}$, and the "first step" is positive temporal direction corresponding to $\hat{U}_{4}$. All the trajectories with the odd-number length $N_{t}$ cannot form a closed loop on the square lattice, and therefore they are gauge-variant and give no contribution in $\left\langle\operatorname{Tr}_{c, \gamma}\left(\hat{U}_{4} \hat{D}^{N_{t}-1}\right)\right\rangle$, except for the Polyakov loop.

Therefore, among the trajectories stemming from $\left\langle\operatorname{Tr}_{c, \gamma}\left(\hat{U}_{4} \hat{D}^{N_{t}-1}\right)\right\rangle$, all the non-loop trajectories are gauge-variant and give no contribution, according to the Elitzur theorem [11]. Only the exception is the Polyakov loop, as shown in Fig.4. (Compare Figs.3 and 4.) Note here that $\left\langle\operatorname{Tr}_{c, \gamma}\left(\hat{U}_{4} \hat{D}^{N_{t}-1}\right)\right\rangle$ do not include the anti-Polyakov loop $\left\langle L_{P}^{\dagger}\right\rangle$, because the "first step" is positive temporal direction corresponding to $\hat{U}_{4}$. 
Polyakov loop

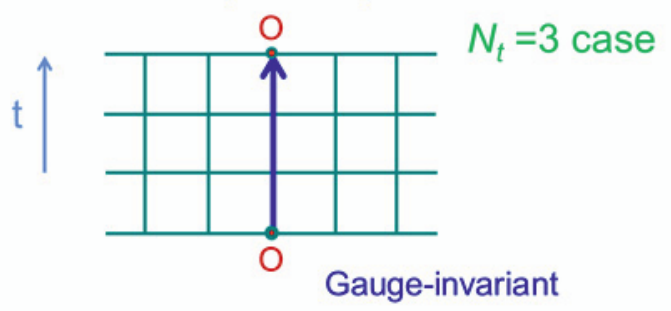

Figure 4. Among the trajectories stemming from $\left\langle\operatorname{Tr}_{c, \gamma}\left(\hat{U}_{4} \hat{D}^{N_{t}-1}\right)\right\rangle$, only the Polyakov-loop ingredient can survive as the gauge-invariant quantity. Here, $\left\langle\operatorname{Tr}_{c, \gamma}\left(\hat{U}_{4} \hat{D}^{N_{t}-1}\right)\right\rangle$ does not include $\left\langle L_{P}^{\dagger}\right\rangle$, because of the first factor $\hat{U}_{4}$.

In this way, only the Polyakov-loop ingredient can survive as the gauge-invariant quantity in the functional trace $\langle I\rangle=\left\langle\operatorname{Tr}_{c, \gamma}\left(\hat{U}_{4} \hat{D}^{N_{t}-1}\right)\right\rangle$, and $\langle I\rangle$ is proportional to the Polyakov loop $\left\langle L_{P}\right\rangle$.

Actually, we can mathematically derive the following relation:

$$
\begin{array}{rlrl}
\langle I\rangle & =\left\langle\operatorname{Tr}_{c, \gamma}\left(\hat{U}_{4} \hat{D}^{N_{t}-1}\right)\right\rangle & \\
& =\left\langle\operatorname{Tr}_{c, \gamma}\left\{\hat{U}_{4}\left(\gamma_{4} \hat{D}_{4}\right)^{N_{t}-1}\right\}\right\rangle & & (\because \text { only gauge-invariant terms survive }) \\
& =4\left\langle\operatorname{Tr}_{c}\left(\hat{U}_{4} \hat{D}_{4}^{N_{t}-1}\right)\right\rangle & & \left(\because \gamma_{4}^{N_{t}-1}=1, \operatorname{tr}_{\gamma} 1=4\right) \\
& =\frac{4}{(2 a)^{N_{t}-1}}\left\langle\operatorname{Tr}_{c}\left\{\hat{U}_{4}\left(\hat{U}_{4}-\hat{U}_{-4}\right)^{N_{t}-1}\right\}\right\rangle & & \left(\because \hat{D}_{4}=\frac{1}{2 a}\left(\hat{U}_{4}-\hat{U}_{-4}\right)\right) \\
& =\frac{4}{(2 a)^{N_{t}-1}}\left\langle\operatorname{Tr}_{c}\left\{\hat{U}_{4}^{N_{t}}\right\}\right\rangle & & (\because \text { only gauge-invariant terms survive }) \\
& =\frac{12 V}{(2 a)^{N_{t}-1}}\left\langle L_{P}\right\rangle .
\end{array}
$$

Thus, we obtain the relation between $\langle I\rangle=\left\langle\operatorname{Tr}_{c, \gamma}\left(\hat{U}_{4} \hat{D}^{N_{t}-1}\right)\right\rangle$ and the Polyakov loop $\left\langle L_{P}\right\rangle$ :

$$
\langle I\rangle=\left\langle\operatorname{Tr}_{c, \gamma}\left(\hat{U}_{4} \hat{D}^{N_{t}-1}\right)\right\rangle=\frac{12 V}{(2 a)^{N_{t}-1}}\left\langle L_{P}\right\rangle .
$$

On the other hand, the functional trace in Eq.(12) can be calculated with the complete set of the Dirac-mode basis $|n\rangle$ satisfying $\sum_{n}|n\rangle\langle n|=1$, and we find the Dirac-mode representation of

$$
\langle I\rangle=\sum_{n}\left\langle n\left|\hat{U}_{4} \hat{D}^{N_{t}-1}\right| n\right\rangle=i^{N_{t}-1} \sum_{n} \lambda_{n}^{N_{t}-1}\left\langle n\left|\hat{U}_{4}\right| n\right\rangle .
$$

By combing Eqs.(14) and (15), we obtain the analytical relation between the Polyakov loop $\left\langle L_{P}\right\rangle$ and the Dirac eigenvalues $i \lambda_{n}$ in QCD:

$$
\left\langle L_{P}\right\rangle=\frac{(2 a i)^{N_{t}-1}}{12 V} \sum_{n} \lambda_{n}^{N_{t}-1}\left\langle n\left|\hat{U}_{4}\right| n\right\rangle .
$$

This is a Dirac spectral representation of the Polyakov loop, and is mathematically valid on the temporally odd-number lattice in both confined and deconfined phases. Based on Eq.(16), we can investigate each Dirac-mode contribution to the Polyakov loop individually, e.g., by evaluating each contribution specified by $n$ numerically in lattice QCD. In particular, by paying attention to low-lying Dirac modes in Eq.(16), the relation between confinement and chiral symmetry breaking can be discussed in QCD. 


\section{Discussions and concluding remarks}

Finally, we discuss the physical meaning of Eq.(16). As a remarkable fact, because of the factor $\lambda_{n}^{N_{t}-1}$, the contribution from low-lying Dirac-modes with $\left|\lambda_{n}\right| \simeq 0$ is negligibly small in the Dirac spectral sum of RHS in Eq.(16), compared to the other Dirac-mode contribution. In fact, the low-lying Dirac modes have quite small contribution to the Polyakov loop, regardless of confined or deconfined phase.

This result is consistent with our previous numerical lattice result that confinement properties are almost unchanged by removing low-lying Dirac modes from the QCD vacuum [6-8].

Here, we give several meaningful comments on the relation (16) in order.

1. Equation (16) is a manifestly gauge-invariant relation. Actually, the matrix element $\left\langle n\left|\hat{U}_{4}\right| n\right\rangle$ can be expressed with the Dirac eigenfunction $\psi_{n}(s)$ and the temporal link-variable $U_{4}(s)$ as

$$
\left\langle n\left|\hat{U}_{4}\right| n\right\rangle=\sum_{s}\langle n \mid s\rangle\left\langle s\left|\hat{U}_{4}\right| s+\hat{t}\right\rangle\langle s+\hat{t} \mid n\rangle=\sum_{s} \psi_{n}^{\dagger}(s) U_{4}(s) \psi_{n}(s+\hat{t})
$$

and each term $\psi_{n}^{\dagger}(s) U_{4}(s) \psi_{n}(s+\hat{t})$ is manifestly gauge invariant, due to the gauge transformation property (5). [Global phase factors also cancel exactly as $e^{-i \varphi_{n}} e^{i \varphi_{n}}=1$ between $\langle n|$ and $|n\rangle$.]

2. In RHS of Eq.(16), there is no cancellation between chiral-pair Dirac eigen-states, $|n\rangle$ and $\gamma_{5}|n\rangle$, because $\left(N_{t}-1\right)$ is even, i.e., $\left(-\lambda_{n}\right)^{N_{t}-1}=\lambda_{n}^{N_{t}-1}$, and $\left\langle n\left|\gamma_{5} \hat{U}_{4} \gamma_{5}\right| n\right\rangle=\left\langle n\left|\hat{U}_{4}\right| n\right\rangle$.

3. Even in the presence of a possible multiplicative renormalization factor for the Polyakov loop, the contribution from the low-lying Dirac modes (or the small $\left|\lambda_{n}\right|$ region) is relatively negligible, compared to other Dirac-mode contribution in the sum of RHS in Eq.(16).

4. For the arbitrary color number $N_{c}$, Eq.(16) is true and applicable in the $\mathrm{SU}\left(N_{c}\right)$ gauge theory.

5. If RHS in Eq.(16) were not a sum but a product, low-lying Dirac modes (or the small $\left|\lambda_{n}\right|$ region) should have given an important contribution to the Polyakov loop as a crucial reduction factor of $\lambda_{n}^{N_{t}-1}$. In the sum, however, the contribution $\left(\propto \lambda_{n}^{N_{t}-1}\right)$ from the small $\left|\lambda_{n}\right|$ region is negligible.

6. Even if $\left\langle n\left|\hat{U}_{4}\right| n\right\rangle$ behaves as $\delta(\lambda)$, the factor $\lambda_{n}^{N_{t}-1}$ is still crucial in Eq.(16), because of $\lambda \delta(\lambda)=0$.

7. The relation (16) is correct regardless of presence or absence of dynamical quarks, although the dynamical quark effect appears in $\left\langle L_{P}\right\rangle$, the Dirac eigenvalue distribution $\rho(\lambda)$ and $\left\langle n\left|\hat{U}_{4}\right| n\right\rangle$.

8. The relation (16) is correct also at finite density and finite temperature.

9. Equation (16) obtained on the odd-number lattice is correct in the continuum limit of $a \rightarrow 0$ and $N_{t} \rightarrow \infty$, since any number of large $N_{t}$ gives the same physical result.

Most of the above arguments can be numerically investigated by lattice QCD calculations. Using actual lattice QCD calculations at the quenched level, we numerically confirm the analytical relation (16), non-zero finiteness of $\left\langle n\left|\hat{U}_{4}\right| n\right\rangle$ for each Dirac mode, and the negligibly small contribution of lowlying Dirac modes to the Polyakov loop, in both confined and deconfined phases [9, 10]. (Although we numerically find an interesting drastic change of the behavior of $\left\langle n\left|\hat{U}_{4}\right| n\right\rangle$ between confined and deconfined phases, we find also tiny contribution of low-lying Dirac modes to the Polyakov loop.)

From the analytical relation (16) and the numerical confirmation, we conclude that low-lying Dirac-modes have quite small contribution to the Polyakov loop, and are not essential for confinement, while these modes are essential for chiral symmetry breaking. This conclusion indicates no direct oneto-one correspondence between confinement and chiral symmetry breaking in QCD. 
It is interesting to compare with other lattice result on importance of infrared gluons to confinement: confinement originates from the low-momentum gluons below $1.5 \mathrm{GeV}$ in Landau gauge [23]. Also, some independence between confinement and chiral symmetry breaking may lead to richer phase structure in QCD, e.g., difference of phase transition points between deconfinement and chiral restoration in strong electro-magnetic fields, due to their nontrivial effect on chiral symmetry [24].

\section{Acknowledgements}

H.S. thanks Prof. K. Redlich and Dr. C. Sasaki for useful discussions. H.S. and T.I. are supported in part by the Grant for Scientific Research [(C) No.23540306, E01:21105006, No.21674002] from the Ministry of Education, Science and Technology of Japan.

\section{References}

[1] Y. Nambu and G. Jona-Lasinio, Phys. Rev. 122, 345 (1961); Phys. Rev. 124, 246 (1961).

[2] H. Suganuma, S. Sasaki and H. Toki, Nucl. Phys. B435, 207 (1995).

H. Suganuma, S. Sasaki, H. Toki and H. Ichie, Prog. Theor. Phys. Suppl. 120, 57 (1995).

[3] O. Miyamura, Phys. Lett. B353, 91 (1995); R.M. Woloshyn, Phys. Rev. D51, 6411 (1995).

[4] C. Gattringer, Phys. Rev. Lett. 97, 032003 (2006).

F. Bruckmann, C. Gattringer and C. Hagen, Phys. Lett. B647, 56 (2007).

[5] F. Synatschke, A. Wipf and K. Langfeld, Phys. Rev. D77, 114018 (2008).

[6] H. Suganuma, S. Gongyo, T. Iritani and A. Yamamoto, PoS (QCD-TNT-II), 044 (2011).

H. Suganuma, S.Gongyo and T.Iritani, PoS (Lattice 2012), 217 (2012).

[7] S. Gongyo, T. Iritani and H. Suganuma, Phys. Rev. D86, 034510 (2012).

[8] T. Iritani and H. Suganuma, arXiv:1305.4049[hep-lat]; T. Iritani, S. Gongyo and H. Suganuma, PoS (Lattice 2012), 218 (2012); PoS (Confinement X), 053 (2013).

[9] H. Suganuma, T.M. Doi and T. Iritani, PoS (Lattice 2013), 374 (2013).

[10] T.M. Doi, H. Suganuma and T. Iritani, PoS (Lattice 2013), 375 (2013).

[11] H.-J. Rothe, Lattice Gauge Theories, 4th edition, (World Scientific, 2012) and its references.

[12] P.M. Lo, B. Friman, O. Kaczmarek, K. Redlich and C. Sasaki, Phys. Rev. D88, 014506 (2013); Phys. Rev. D88, 074502 (2013).

[13] T. Banks and A. Casher, Nucl. Phys. B169, 103 (1980).

[14] F. Karsch, Lect. Notes Phys. 583, 209 (2002), and its references.

[15] Y. Aoki, Z. Fodor, S.D. Katz and K.K. Szabo, Phys. Lett. B643, 46 (2006).

[16] A.S. Kronfeld, G. Schierholz and U.-J. Wiese, Nucl. Phys. B293, 461 (1987).

[17] J.D. Stack, S.D. Neiman and R.J. Wensley, Phys. Rev. D50, 3399 (1994).

[18] K. Amemiya and H. Suganuma, Phys. Rev. D60, 114509 (1999).

S. Gongyo, T. Iritani and H. Suganuma, Phys. Rev. D86, 094018 (2012).

S. Gongyo and H. Suganuma, Phys. Rev. D87, 074506 (2013).

[19] H. Ichie and H. Suganuma, Nucl. Phys. B574, 70 (2000); Nucl. Phys. B548, 365 (1999).

[20] Y. Nambu, Phys. Rev. D10, 4262 (1974); G. 't Hooft, Nucl. Phys. B190, 455 (1981).

[21] H. Suganuma, A. Tanaka, S. Sasaki and O. Miyamura, Nucl. Phys. Proc. Suppl. 47, 302 (1996).

[22] C.B. Lang and M. Schrock, Phys. Rev. D84, 087704 (2011).

L. Ya Glozman, C.B. Lang and M. Schrock, Phys. Rev. D86, 014507 (2012).

[23] A.Yamamoto, H.Suganuma, Phys.Rev.Lett.101, 241601 (2008); Phys.Rev.D79, 054504 (2009).

[24] H. Suganuma and T. Tatsumi, Ann. Phys. 208, 470 (1991); Prog. Theor. Phys. 90, 379 (1993). 